\title{
Avaliação da satisfação sexual de homens atendidos em ambulatório de urologia
}

\author{
Evaluation of the sexual satisfaction of men attended in urology outpatient clinic \\ Evaluación de la satisfacción sexual de hombres atendidos en ambulatorio de urología
}

Alexandre Franco do Val Serafim ${ }^{1}$, Raílson Cavalcante Silva ${ }^{1}$, Lucas Cristovam Pina ${ }^{1}$, Antonio Márcio Teodoro Cordeiro Silva ${ }^{1}$, Rogério José de Almeida ${ }^{1 *}$

\begin{abstract}
RESUMO
Objetivo: Avaliar a satisfação sexual em homens atendidos em serviço de urologia de um hospital escola da cidade de Goiânia/GO. Métodos: Trata-se de um estudo transversal analítico com abordagem quantitativa. A pesquisa foi realizada por meio de questionários aplicados em 91 homens atendidos no ambulatório de urologia do hospital. Foram utilizados dois instrumentos, sendo um sociodemográfico e clínico, o outro a Escala de Satisfação Sexual Golombok e Rust (GRISS) versão masculina. Foi realizada estatística descritiva, Teste exato de Fisher e Teste G. Para todos os testes comparativos foi assumido $p$-valor menor ou igual a 0,05 como significativo. Resultados: Identificou-se que a satisfação sexual não está relacionada apenas as disfunções orgânicas e sim a fatores biopsicossociais. O presente estudo identificou alguns importantes fatores associados a uma baixa satisfação sexual masculina, são eles: ter mais que 60 anos, ser viúvo, não ter uma religião, ter rendimento pessoal entre um e quatro salários mínimos, não ter lazer frequente, não fazer uso de internet frequentemente, ensino superior completo/incompleto, presença de doença respiratória e uso de bebida alcóolica. Conclusão: A satisfação sexual é de fundamental importância para o bem-estar do ser humano. Observa-se que sua estruturação envolve aspectos biológicos, culturais, sociais e emocionais. Este estudo detectou uma piora da função sexual nos homens idosos e as questões que envolvem seu estilo de vida. Esse é um grande desafio, haja visa que o aumento da expectativa de vida da população masculina propiciadas pelos avanços tecnológicos e medidas preventivas é um fato.
\end{abstract}

Palavras-chave: Saúde do homem; Saúde sexual; Urologia.

\begin{abstract}
Objective: To evaluate sexual satisfaction in men attending a urology service at a school hospital in the city of Goiânia/GO. Methods: This is an analytical cross-sectional study with a quantitative approach. The research was carried out through questionnaires applied to 91 men attended at the urology outpatient clinic of the hospital. Two instruments were used, one socio-demographic and clinical, the other the Golombok and Rust Sexual Satisfaction Scale (GRISS) male version. Descriptive statistics, Fisher's exact test and G-test were performed. For all comparative tests $p$-value less than or equal to 0.05 was assumed as significant. Results: It was identified that sexual satisfaction is related not only to organic dysfunctions but to biopsychosocial factors. The present study identified some important factors associated with low male sexual satisfaction: they are over 60 years of age, widowed, have no religion, have a personal income between one and four minimum wages, do not have frequent leisure, do not use of internet frequently, complete / incomplete higher education, the presence of respiratory disease and the use of alcoholic beverage. Conclusion: Sexual satisfaction is of fundamental importance for the well-being of the human being. It is observed that its structuring involves biological, cultural, social and emotional aspects. This study detected a worsening of
\end{abstract}

${ }^{1}$ Pontifícia Universidade Católica de Goiás - PUC Goiás. * E-mail: rogeriopucgo@gmail.com 
sexual function in older men and the issues surrounding their lifestyle. This is a great challenge, since it aims at increasing the life expectancy of the male population brought about by technological advances and preventive measures is a fact.

Palavras-chave: Men's health; Sexual health; Urology.

\section{RESUMEN}

Objetivo: Evaluar la satisfacción sexual en hombres atendidos en servicio de urología de un hospital escuela de la ciudad de Goiânia/GO. Métodos: Se trata de un estudio transversal analítico con abordaje cuantitativo. La investigación fue realizada por medio de cuestionarios aplicados en 91 hombres atendidos en el ambulatorio de urología del hospital. Se utilizaron dos instrumentos, siendo un sociodemográfico y clínico, el otro la Escala de Satisfacción Sexual Golombok y Rust (GRISS) versión masculina. Se realizó una estadística descriptiva, Prueba exacta de Fisher y Test G. Para todas las pruebas comparativas se asumió p-valor menor o igual a 0,05 como significativo. Resultados: Se identificó que la satisfacción sexual no está relacionada sólo con las disfunciones orgánicas y sí a factores biopsicosociales. El presente estudio identificó algunos importantes factores asociados a una baja satisfacción sexual masculina, son ellos: tener más de 60 años, ser viudo, no tener una religión, tener rendimiento personal entre uno y cuatro salarios mínimos, no tener ocio frecuente, no hacer uso de Internet a menudo, enseñanza superior completa/incompleta, presencia de enfermedad respiratoria y uso de bebida alcohólica. Conclusión: La satisfacción sexual es de fundamental importancia para el bienestar del ser humano. Se observa que su estructuración involucra aspectos biológicos, culturales, sociales y emocionales. Este estudio detectó un empeoramiento de la función sexual en los hombres mayores y las cuestiones que involucran su estilo de vida. Este es un gran desafío, ya que el aumento de la expectativa de vida de la población masculina propiciada por los avances tecnológicos y medidas preventivas es un hecho.

Palabras clave: Salud del hombre; Salud sexual; Urología.

\section{INTRODUÇÃO}

O desempenho sexual é de fundamental importância para o bem-estar do ser humano. Este envolve características da personalidade de cada indivíduo (BRITTO; BENETTI, 2010). Trata-se de um processo contínuo da evolução do ser, que se inicia desde a vida embrionária com a diferenciação sexual até findar com a morte do indivíduo. Sua estruturação envolve aspectos biológicos, culturais, sociais, emocionais, sentimentais, crenças e religião na qual estão relacionados entre si para a construção da sexualidade (BYERS, 2011).

De acordo com esses aspectos biopsicossociais, o desenvolvimento da sexualidade se dá de forma individual e peculiar podendo ser alterado de acordo com as experiências e modificações orgânicas ao longo da vida (GALATI et al., 2014). Para se obter uma satisfação sexual deve haver harmonia entre essa complexidade de fatores (fisiológicos, comportamentais e culturais), conduz assim a um bem-estar psicológico do ser humano (DOMINGOS, 2013).

Assim sendo, a questão da função sexual é de suma importância, podendo ser influenciada tanto de forma positiva, melhora do bem-estar do indivíduo, autoconfiança, elevação da autoestima, melhora das relações interpessoais e conjugal, quanto de uma forma negativa, com angústia, sentimento de frustração, baixa autoestima e impotência por não conseguir realizar de forma satisfatória o ato sexual, ponto-chave para um quadro de disfunção sexual (GALATI et al.,, 2014). 
Segundo o Manual Diagnóstico e Estatístico de Transtornos Mentais - DSM V (2014), o transtorno sexual caracteriza-se por uma perturbação clinicamente significativa na capacidade de uma pessoa responder sexualmente ou de experimentar prazer sexual. Pode ter no mesmo indivíduo disfunções sexuais diferentes como ejaculação precoce ou retardada, disfunção erétil, desejo sexual hipoativo, entre outros.

Assim, a disfunção sexual caracteriza-se por qualquer problema que interfira nas etapas que compõem o ato sexual do indivíduo. Segundo Galati et al. (2014), grande parte dos homens sofrem algum comprometimento nessas fases, como por exemplo, pode haver uma excitação inadequada, o que pode resultar na incapacidade de ereção e dificuldade para atingir o clímax sexual. São alterações que podem interferir na percepção que o homem tem do seu prazer. Mesmo nos casos em que não se relacionam à disfunção, a ansiedade geralmente acompanha a maioria dos casos e deve ser considerada na abordagem diagnóstica e terapêutica, pois pode prolongar e exacerbar os problemas existentes (LUCENA; ABDO, 2013).

Com aumento da expectativa de vida, a discussão a respeito de disfunção sexual dos homens ganhou mais enfoque, principalmente com a descoberta do Viagra. Este é um fármaco que promoveu um prolongamento na vida sexual masculina, que atua de forma direta e benéfica na disfunção erétil. Com aumento da longevidade desta população, os avanços tecnológicos a favor da medicina propiciaram uma atividade sexual estendida por mais anos (SARRIS et al., 2016).

Se há um problema na função sexual, logo há também na satisfação sexual, a qual é considerada um indicador de saúde representando um resultado positivo da atividade sexual associado ao bem-estar geral, à saúde individual e ao ajustamento relacional (BYERS, 2011). Nesse sentido, o foco da análise da problematização empreendida situa-se na avaliação da satisfação sexual em homens e teve por objetivo analisar a satisfação sexual em homens atendidos em serviço de urologia de um Hospital Escola da cidade de Goiânia/GO.

\section{MÉTODOS}

Trata-se de um estudo transversal analítico com abordagem quantitativa. Este é um método de pesquisa que investiga a ocorrência de agravos à saúde, assim como a associação entre eles a partir de observações pontuais no tempo estabelecido satisfazendo assim o presente estudo (ARAGÃO, 2011). A medida de ocorrência deste tipo de estudo é estruturada a partir da prevalência na qual poderá relacionar tais varáveis com a satisfação sexual masculina investigada em um determinado período.

A pesquisa foi realizada por meio de questionários aplicados aos homens atendidos nos ambulatórios de Urologia de um Hospital Escola da cidade de Goiânia/GO. Foram pesquisados pacientes atendidos no ambulatório de urologia do referido hospital que foram encaminhados via regulação da Secretaria Municipal de Goiânia e estadual de Goiás.

Os critérios de inclusão foram pacientes do sexo masculino, faixa etária acima dos 18 anos, com queixas de patologias com interferência direta ou indiretamente na satisfação sexual. Foram excluídos do estudo pacientes com déficit cognitivo, fora da faixa etária estabelecida e que não responderam todas as questões dos questionários.

Para a realização do cálculo amostral foi utilizado o número de pacientes atendidos nos últimos dois meses (fevereiro e março de 2018) que antecederam a coleta de dados. Nesse período, foram atendidos, dentro dos critérios de inclusão e exclusão, um total de 117 pacientes. Com esta população utilizou-se um nível de confiança de $95 \%$ e margem de erro de $5 \%$, com a amostra final composta por 90 pacientes, sendo utilizada uma amostragem por conveniência durante os meses de abril e maio de 2018.

Para o desenvolvimento desta pesquisa, foi utilizado um questionário epidemiológico que foi construído pelos autores com o objetivo de traçar o perfil sociodemográfico e clínico dos participantes. Abrange variáveis como idade, estado civil, cor ou etnia autorreferida, escolaridade, religião, renda pessoal, práticas de atividade física, se possuem doenças crônicas, utilização de medicamentos contínuos ou para melhorar sua satisfação sexual, se é uma pessoa ansiosa ou triste, se faz ingesta de bebida alcóolica, tabagista.

REAS/EJCH | Vol. 11 (4) | e298 | DOI: https://doi.org/10.25248/reas.e298.2019 Página 3 de 9 
Utilizou-se também a Escala de Satisfação Sexual Golombok e Rust (GRISS) versão masculina. A GRISS foi inicialmente desenvolvida por Golombok e Rust no ano de 1986. Permite avaliar a satisfação sexual e é constituída por duas versões, uma para o sexo feminino e outra para o masculino (GOLOMBOK; RUST, 1986).

Sua tradução e adaptação para o Brasil foi realizada em uma pesquisa com 626 sujeitos do Estado do Rio Grande do Sul (339 mulheres e 287 homens), com idades variando entre 18 e 74 anos (DIEHL, 2000). Este inventário é composto por 28 questões, respondidas segundo uma escala tipo Likert que varia de 0 "nunca" e 4 "sempre" (DIEHL, 2000; GOLOMBOK; RUST, 1986).

A somatória dos pontos atingidos é transformada em um único valor, denominado escore global, com variação entre 1 e 9 . Os valores a partir de 5 indicam a ocorrência de problemas com a satisfação sexual. Quanto mais elevada for a pontuação atingida, menor é a qualidade do funcionamento sexual e/ou maior é o problema ou a dificuldade sexual (DIEHL, 2000).

Com os dados coletados foi confeccionado um banco de dados utilizando o software IBM SPSS Statistics 18. Posteriormente, foi realizada estatística descritiva com o cálculo de frequências absoluta e relativa percentual. Na sequência, foi aplicado teste de normalidade (Kolmogorov-Smirnov) para distinguir as distribuições paramétricas e não-paramétricas, com o intuito de comparação dos resultados do questionário estratificado pelas variáveis sociodemográficas e clínicas. Sendo assim, foram utilizados os seguintes testes estatísticos: Teste exato de Fisher e Teste G. Para todos os testes comparativos foi assumido p-valor menor ou igual a 0,05 como significativo.

A pesquisa foi registrada na Plataforma Brasil do Ministério da Saúde, aprovada pelo Comitê de Ética em Pesquisa da Pontifícia Universidade Católica de Goiás - PUC Goiás com o parecer no 2.404.432 e pelo Comitê de Ética em Pesquisa do Hospital Santa Casa de Misericórdia de Goiânia com o parecer no 2.519.072.

\section{RESULTADOS}

Foram pesquisados um total de 90 homens que foram atendidos em ambulatório de urologia de um hospital escola da cidade de Goiânia GO. Dentre esses, 57,8\% tinham menos que 60 anos e 42,2\% eram idosos com 60 anos ou mais de idade. A maioria era de casados ou em união estável $(61,1 \%)$, com ensino fundamental $(38,9 \%)$ e ensino médio $(38,9 \%)$, com renda pessoal de um a quatro salários mínimos $(74,4 \%)$, e que referiram a utilização frequente da internet $(70,0 \%)$ (Tabela 1$)$.

Os escores da escala GRISS identificaram que $(62,2 \%)$ não apresentaram problemas em relação à satisfação sexual e, ao contrário, $(27,8 \%)$ homens apresentaram escore igual ou acima de cinco enquadrando-se nos casos com problemas com a satisfação sexual (Tabela 1).

$\mathrm{Na}$ análise comparativa da satisfação sexual medida pela Escala GRISS com os aspectos sociodemográficos, identificou-se com significância estatística que os homens com 60 anos ou mais de idade, ou seja, os idosos apresentaram maiores escores indicando uma baixa satisfação sexual $(p<0,0001)$. $O$ estado civil "viúvo" apareceu como um forte preditor para a insatisfação sexual masculina $(p<0,0001)$. Outro dado importante é que quanto menor a escolaridade, maiores são os problemas com a função sexual dos homens entrevistados $(p<0,0001)$ (Tabela 1).

O fato ser religioso $(p=0,025)$, rendimento pessoal entre um e quatro salários mínimos $(p=0,013)$, não ter lazer frequente $(p=0,004)$ e não utilizar a internet frequentemente $(p<0,0001)$ foram identificados como preditores de baixa satisfação sexual (Tabela 1).

$\mathrm{Na}$ análise comparativa da satisfação sexual medida pela Escala GRISS com os aspectos clínicos identificou-se com significância estatística que ter doença respiratória $(p=0,048)$, não fazer uso de álcool $(p=0,0002)$ (Tabela 2). 
Tabela 1. Comparação da satisfação sexual medida pela Escala GRISS com os aspectos sociodemográficos de 90 homens atendidos em serviço de urologia, Goiânia, Goiás, Brasil, 2018.

\begin{tabular}{|c|c|c|c|c|c|c|c|}
\hline \multirow{3}{*}{ Variável $(n=90)$} & \multirow{3}{*}{$n$} & \multicolumn{6}{|c|}{ GRISS } \\
\hline & & \multirow[t]{2}{*}{$f(\%)$} & \multicolumn{2}{|c|}{ Escore $<5(n=65)$} & \multicolumn{2}{|c|}{ Escore $\geq 5(n=25)$} & \multirow[t]{2}{*}{$p$-valor } \\
\hline & & & $n$ & $f(\%)$ & $n$ & $f(\%)$ & \\
\hline \multicolumn{8}{|l|}{ Idade (anos) } \\
\hline$<60$ & 52 & 57,8 & 48 & 73,8 & 4 & 16,0 & \\
\hline$\geq 60$ & 38 & 42,2 & 17 & 26,2 & 21 & 84,0 & $<0,0001$ \\
\hline \multicolumn{8}{|l|}{ Estado Civil } \\
\hline Solteiro & 15 & 16,7 & 12 & 18,5 & 3 & 12,0 & \\
\hline Casado/União Estável & 55 & 61,1 & 42 & 64,6 & 13 & 52,0 & \\
\hline Divorciado & 12 & 13,3 & 11 & 16,9 & 1 & 4,0 & \\
\hline Viúvo & 8 & 8,9 & 0 & 0,0 & 8 & 32,0 & $<0,0001$ \\
\hline \multicolumn{8}{|l|}{ Cor ou Raça Autorreferida } \\
\hline Branca & 43 & 47,8 & 31 & 47,7 & 12 & 48,0 & \\
\hline Preta & 18 & 20,0 & 11 & 16,9 & 7 & 28,0 & \\
\hline Amarela & 4 & 4,4 & 3 & 4,6 & 1 & 4,0 & \\
\hline Parda & 24 & 26,7 & 20 & 30,8 & 4 & 16,0 & \\
\hline Indígena & 1 & 1,1 & 0 & 0,0 & 1 & 4,0 & 0,263 \\
\hline \multicolumn{8}{|l|}{ Escolaridade } \\
\hline Não sabe ler e nem escrever & 7 & 7,8 & 1 & 1,5 & 6 & 24,0 & \\
\hline Ensino fundamental & 35 & 38,9 & 19 & 29,2 & 16 & 64,0 & \\
\hline Ensino médio & 35 & 38,9 & 32 & 49,2 & 3 & 12,0 & \\
\hline Superior Completo/Incompleto & 13 & 14,4 & 13 & 20,0 & 0 & 0,0 & $<0,0001$ \\
\hline \multicolumn{8}{|l|}{ Religião } \\
\hline Sim & 80 & 88,9 & 61 & 93,8 & 19 & 76,0 & \\
\hline Não & 10 & 11,1 & 4 & 6,2 & 6 & 24,0 & 0,025 \\
\hline \multicolumn{8}{|l|}{ Rendimento Pessoal } \\
\hline Abaixo de um salário mínimo & 11 & 12,2 & 8 & 12,3 & 3 & 12,0 & \\
\hline De 1 a 4 salários mínimos & 67 & 74,4 & 45 & 69,2 & 22 & 88,0 & \\
\hline Acima de 4 salários mínimos & 12 & 13,3 & 12 & 18,5 & 0 & 0,0 & 0,013 \\
\hline \multicolumn{8}{|l|}{ Lazer Frequente } \\
\hline Sim & 45 & 50,0 & 39 & 60,0 & 6 & 24,0 & \\
\hline Não & 45 & 50,0 & 26 & 40,0 & 19 & 76,0 & 0,004 \\
\hline \multicolumn{8}{|l|}{ Internet com Frequência } \\
\hline Sim & 63 & 70,0 & 56 & 86,2 & 7 & 28,0 & \\
\hline Não & 27 & 30,0 & 9 & 13,8 & 18 & 72,0 & $<0,0001$ \\
\hline \multicolumn{8}{|l|}{ Atividade Física } \\
\hline Sim & 46 & 51,1 & 37 & 56,9 & 9 & 36,0 & \\
\hline Não & 44 & 48,9 & 28 & 43,1 & 16 & 64,0 & 0,100 \\
\hline
\end{tabular}

Fonte: Dados da pesquisa, 2018. 
Tabela 2. Comparação da satisfação sexual medida pela Escala GRISS com os aspectos clínicos de 90 homens atendidos em serviço de urologia, Goiânia, Goiás, Brasil, 2018.

\begin{tabular}{|c|c|c|c|c|c|c|c|}
\hline \multirow{3}{*}{ Variável $(n=90)$} & \multirow{3}{*}{$n$} & \multicolumn{6}{|c|}{ GRISS } \\
\hline & & \multirow[t]{2}{*}{$f(\%)$} & \multicolumn{2}{|c|}{ Escore $<5(n=65)$} & \multicolumn{2}{|c|}{ Escore $\geq 5(n=25)$} & \multirow[t]{2}{*}{$p$-valor } \\
\hline & & & $n$ & $f(\%)$ & $n$ & $f(\%)$ & \\
\hline \multicolumn{8}{|l|}{ Doença Crônica } \\
\hline Sim & 42 & 46,7 & 28 & 43,1 & 14 & 56,0 & \\
\hline Não & 48 & 53,3 & 37 & 56,9 & 11 & 44,0 & 0,347 \\
\hline \multicolumn{8}{|c|}{ Doença Neurológica } \\
\hline Sim & 3 & 3,3 & 1 & 1,5 & 2 & 8,0 & \\
\hline Não & 87 & 96,7 & 64 & 98,5 & 23 & 92,0 & 0,186 \\
\hline \multicolumn{8}{|c|}{ Doença Respiratória } \\
\hline Sim & 6 & 6,7 & 2 & 3,1 & 4 & 16,0 & \\
\hline Não & 84 & 93,3 & 63 & 96,9 & 21 & 84,0 & 0,048 \\
\hline \multicolumn{8}{|c|}{ Doença Cardíaca } \\
\hline Sim & 29 & 32,2 & 20 & 30,8 & 9 & 36,0 & \\
\hline Não & 61 & 67,8 & 45 & 69,2 & 16 & 64,0 & 0,802 \\
\hline \multicolumn{8}{|c|}{ Usa Medicamento } \\
\hline Sim & 45 & 50,0 & 28 & 43,1 & 17 & 68,0 & \\
\hline Não & 45 & 50,0 & 37 & 56,9 & 8 & 32,0 & 0,059 \\
\hline \multicolumn{8}{|c|}{ Medicamento para sexo } \\
\hline $\operatorname{Sim}$ & 23 & 25,6 & 19 & 29,2 & 4 & 16,0 & \\
\hline Não & 67 & 74,4 & 46 & 70,8 & 21 & 84,0 & 0,282 \\
\hline \multicolumn{8}{|c|}{ Considera-se estressado/ansioso } \\
\hline Sim & 33 & 36,7 & 27 & 41,5 & 6 & 24,0 & \\
\hline Não & 57 & 63,3 & 38 & 58,5 & 19 & 76,0 & 0,148 \\
\hline \multicolumn{8}{|c|}{ Considera-se triste } \\
\hline $\operatorname{Sim}$ & 11 & 12,2 & 6 & 9,2 & 5 & 20,0 & \\
\hline Não & 79 & 87,8 & 59 & 90,8 & 20 & 80,0 & 0,279 \\
\hline \multicolumn{8}{|c|}{ Uso de Álcool Atualmente } \\
\hline Sim & 35 & 38,9 & 33 & 50,8 & 2 & 8,0 & \\
\hline Não & 55 & 61,1 & 32 & 49,2 & 23 & 92,0 & 0,0002 \\
\hline \multicolumn{8}{|l|}{ Fumante } \\
\hline $\operatorname{Sim}$ & 17 & 18,9 & 11 & 16,9 & 6 & 24,0 & \\
\hline Não & 73 & 81,1 & 54 & 83,1 & 19 & 76,0 & 0,549 \\
\hline \multicolumn{8}{|l|}{ Dorme bem } \\
\hline Sim & 73 & 81,1 & 56 & 86,2 & 17 & 68,0 & \\
\hline Não & 17 & 18,9 & 9 & 13,8 & 8 & 32,0 & 0,051 \\
\hline
\end{tabular}

Fonte: Dados da pesquisa, 2018. 


\section{DISCUSSÃO}

No presente estudo, realizado com 90 homens acima de 18 anos, foram identificados que 27,8\% apresentaram problemas em relação à satisfação sexual. Em uma pesquisa realizada no Brasil, Britto e Benetti (2010) identificaram que dos 42 homens com idade entre 12 e 45 anos diagnosticados com disfunção sexual pesquisados, $50 \%$ possuíam disfunção erétil e $50 \%$ tinham ejaculação precoce. Foram observadas uma alta incidência de sintomas depressivos e ansiosos relacionados aos transtornos sexuais, o que mostra que fatores orgânicos e emocionais estavam fortemente associados e não deviam ser tratados de forma isolada.

Em um estudo realizado com 137 homens iranianos com queixas sexuais, os pesquisadores constataram que os problemas relacionados às disfunções sexuais causavam, além da depressão, efeitos negativos sobre a autoimagem, perda da confiança, estresse afetando a saúde em geral com piora da qualidade de vida desses pacientes (NAEINIAN; SHAIERI; HOSSEINI, 2011).

Identificou-se, com significância estatística, que os homens com 60 anos ou mais de idade apresentaram maiores escores, indicando uma baixa satisfação sexual. A sexualidade nos idosos ainda é vista de forma preconceituosa, com frequência associa-se a construção de estereótipos, na qual é destinada apenas aos jovens com uma certa inibição por parte dos idosos em suas relações e vontades sexuais (CIOSAK, 2011). Outros fatores que podem ser associados seriam a presença de doenças crônicas nessa faixa etária em que podem afetar diretamente seus desejos sexuais em decorrência de problemas orgânicos que interferem na fisiologia do organismo, com prejuízo no desempenho sexual.

O estado civil "viúvo" apareceu como um forte preditor para a insatisfação sexual masculina, o que foi observado no estudo nacional de Moreira Jr. et al. (2004), o qual participaram 71.503 viúvos, sendo observado nessa população altos índices de baixa satisfação sexual. A perda de suas companheiras no decorrer dos anos impacta diretamente em sua qualidade de vida, pois se sentem "sozinhos" e sem perspectiva para uma nova relação, e com isso perdem a vontade e o desejo sexual (MOREIRA JR. et al., 2004).

Foi evidenciado, no estudo, que o fato de não ter um lazer frequente contribuiu para uma baixa satisfação sexual. Atualmente, a prática de lazer por parte da população encontra-se cada vez mais infrequente devido a falta de opção ou mesmo pelas limitações físicas, alta carga horária de trabalho, violência que impede o indivíduo de sair de casa, restrição financeira, entre outros que acabam interferindo de maneira direta na qualidade de vida da população com impacto negativo na satisfação sexual.

O fato de os pacientes não seguirem algum tipo de religião, atua de forma negativa na satisfação sexual em relação aos religiosos. Já no estudo realizado por Holanda et al. (2014) mostrou que os dogmas religiosos exercem efeito negativo nas relações sexuais, pois o mesmo não pode desfrutar livremente do prazer sexual e erótico. Já outros buscam a religião como uma forma de interação social e acabam seguindo as doutrinas por elas impostas, na qual as relações sexuais em alguns casos somente são permitidas após o matrimônio, a adesão a essas práticas podem interferir negativamente ou não, indo de acordo com a consciência do indivíduo e a depender dos dogmas religiosos por eles seguidos.

Outro fator que obteve significância estatística foi a presença de doença respiratória associada a uma pior satisfação sexual. A prática do sexo equivale a uma atividade física, na qual demanda um alto gasto energético e necessita de uma troca gasosa adequada nos alvéolos pulmonares. Na presença da patologia respiratória a hematose fica comprometida, com interferência direta na performance adequada, o que contribui negativamente no desempenho sexual, pois, não conseguem obter uma relação adequada que pode cursar com uma certa frustração, e insatisfação por parte desses pacientes (SARRIS et al., 2016).

No presente estudo, o uso de álcool não obteve significância estatística quanto a satisfação sexual, indo em desencontro com o estudo de Lourenço e Fernandes (2011), no qual o consumo abusivo de álcool é algo devastador na vida do casal, pois provoca estresse psicológico, violência e padrões de comunicações disfuncionais que podem gerar hostilidade, conflitualidade e tensão no relacionamento. Com isso, ocorre maus tratos, abandono e negligência, consequentemente uma queda na frequência das relações sexuais. 
Outro ponto que mostrou significância estatística diz respeito ao acesso a internet, na qual homens que não possuem contato com essa tecnologia têm maior insatisfação sexual. Segundo Hintz (2001), a internet pode contribuir de forma negativa, pois o seu uso de maneira exagerada em redes sociais e aplicativos causam o afastamento da convivência conjugal, uma vez que o parceiro sempre conectado e envolvido em tudo que essa tecnologia oferece, podem surgir diferenças de valores e interesses, o que leva o casal a viver distanciado um do outro. $O$ fato do homem buscar satisfação sexual por meio da internet configura-se assim como uma "infidelidade virtual".

Por meio da análise estatística no presente estudo, foi observado que quanto maior o nível de escolaridade menor é a satisfação sexual. Isso se deve ao fato que atualmente vive-se em uma sociedade competitiva, na qual precisa qualificar-se cada vez mais para se destacar no mercado de trabalho. Devido a esse fato, as pessoas dedicam mais tempo aos ensinos superiores, pós-graduações, mestrados, doutorados, entre outros, deixando de lado assim as relações sociais para se dedicar a carreira profissional (HINTZ, 2001). Segundo Nelas et al. (2018), a motivação sexual vai além do instinto, constitui algo hormonal, cognitivo e educacional, na qual quanto maior o nível escolar maiores as chances de relações interpessoais, o indivíduo se torna mais atrativo.

O presente trabalho evidenciou que pacientes que ganham acima de 4 salários mínimos têm maior insatisfação sexual comparados aqueles que têm baixa renda, o que não foi visto no estudo realizado por Kalckmann et al. (2005) em que os homens com menor ganho salarial ou desempregados possuem menor satisfação sexual, pois tal situação traz uma desarmonia conjugal, com interferência direta nas relações interpessoais, na qual afeta assim a satisfação sexual, o que mostra que a relação sexual é algo que vai além do ato fisiológico, com envolvimento também fatores comportamentais e culturais (DOMINGOS, 2013).

Segundo Azevedo et al. (2013), alguns fatores interferem na qualidade de vida, assim como sexo, idade, hábitos prejudiciais à saúde, fumo, consumo de álcool, estado civil e pobreza. Pessoas com doenças crônicas, como as respiratórias, renda mínima e baixa escolaridade têm uma pior qualidade de vida, dentre elas a satisfação sexual.

\section{CONCLUSÃO}

É fato que os homens têm dificuldade em procurar um serviço médico devido a vergonha de falar sobre desordem sexual, por trazer um certo constrangimento e o mesmo se sentir inibido quanto ao assunto envolvido, e como consequência, a oferta de sua doença pode não ser feita de maneira adequada. $A$ dificuldade no questionamento dessas desordens sexuais dá-se pela falta de conhecimento específico do assunto pelos médicos que visam em geral o encaminhamento para o especialista. Sendo assim, pode ser vista como um indicador de saúde e de difícil abordagem nas consultas médicas.

As conclusões chamam a atenção para que o médico tenha conhecimentos e habilidades para mostrar que a fragilidade e a vulnerabilidade não implicam em uma submissão masculina, devem ser tratadas de uma forma correta, passando confiança ao paciente que ali se encontra. A satisfação sexual em qualquer idade deve ser reconhecida como um fenômeno intrínseco ao homem e, como tal, deve ser abordada no encontro clínico.

\section{REFERÊNCIAS}

1. AMERICAN PSYCHIATRIC ASSOCIATION. Manual diagnóstico e estatístico de transtornos mentais: DSM-5. American Psychiatric Association, 2014.

2. ARAGÃO J. Introdução aos estudos quantitativos utilizados em pesquisas científicas. Revista Práxis, 2011; ano III, 6.

3. AZEVEDO ALS, SILVA RA, TOMASI E, et al. Doenças crônicas e qualidade de vida na atenção primária à saúde. Cadernos de saúde pública, 2013; 29(9):1774-82.

4. BRITTO R, BENETTI SPC. Ansiedade, depressão e característica de personalidade em homens com disfunção sexual. Revista da SBPH, 2010; 13(2):243-58.

5. BYERS ES. Sexual satisfaction in romantic relationships: findings from 25 years of research. Conferência sobre Satisfação Sexual, Faculdade de Psicologia de Lisboa, Universidade de Lisboa, 2011.

6. CIOSAK IS, BRAZ E, COSTA MFBNA, et al. Senescência e senilidade: novo paradigma na atenção básica de saúde. Revista da

REAS/EJCH | Vol. 11 (4) | e298 | DOI: https://doi.org/10.25248/reas.e298.2019 Página 8 de 9 
Escola de Enfermagem da USP, 2011; 45(2):1763-68.

7. DIEHL JA. A qualidade do funcionamento sexual de homens e mulheres. Dissertação de Mestrado. Pontifícia Universidade Católica do Rio Grande do Sul, Porto Alegre, 2000.

8. DOMINGOS VGM, BRITTO IAGS. Disfunção sexual masculina: algumas implicações. Fragmentos de Cultura, 2013; 23(4):579-586.

9. EUFRÁSIO JJG, NÓBREGA TP. Representações do corpo masculino na revista Men's Health. Revista Brasileira de Ciência do Esporte, 2017; 39(1):31-38.

10. FINOTELLI JR. I, CAPITÃO CG. Evidências de validade da versão brasileira da Escala de Autoeficácia Sexual - Função Erétil. PsicoUSF, 2011; 16(1):45-55.

11. GALATI MCR, ALVES JR. EO, DELMASCHIO CC, et al. Sexualidade masculina e qualidade de vida em homens com dificuldades sexuais. Psico-USF, 2014; 19(2):243-252.

12. GOLOMBOK S, RUST J. The GRISS: a psychometric instrument for the assessment of sexual dysfunction. Archives of Sexual Behavior, 1986; 15(2):157-65.

13. GOMES R, SCHRAIBER LB, COUTO MT, et al. O atendimento à saúde dos homens: estudo qualitativo em quatro estados brasileiros. Physis: Revista de Saúde Coletiva, 2011; 21(1):113-128.

14. HINTZ HC. Novos tempos, novas famílias? Da modernidade à pós modernidade. Pensando famílias, 2001, 3:8-19.

15. HOFFMAN BL, SCHORGE JO, SCHAFFER JI. Ginecologia de Williams. 2. ed. Porto Alegre: AMGH, 2014.

16. HOLANDA JBL, ABUCHAIM ESV, COCA KP, et al. Disfunção sexual e fatores associados e relatados no período pós-parto. Acta Paulista de Enfermagem, 2014; 27(6):573-8.

17. KALCKMANN S, BATISTA LE, SOUZA LCF. Homens de baixa renda falam sobre saúde reprodutiva e sexual. In: ADORNO R, ALVARENGA A, VASCONCELOS MP (orgs.). Jovens, trajetória, masculinidades e direitos. São Paulo: Edusp; 2005. p. 199-217.

18. KERN CAR. Disfunção sexual masculina: compreensão psicanalítica. Dissertação de Mestrado. Universidade do Vale do Rio dos Sinos, São Leopoldo, RS, 2010.

19. LOURENÇO SM, FERNANDES OM. O álcool entre nós: o impacto do consumo de álcool no casal. Revista CES Psicologia, 2011; $4(2): 15-28$.

20. LUCENA BB, ABDO CHN. O papel da ansiedade na (dis) função sexual. Diagnóstico e tratamento, 2013; 18(2):94-8.

21. MASTERS WH, JOHNSON VE. Human sexual response. Boston: Lippincott Williams e Wilkins, 1966.

22. MOREIRA JR ED, SANTOS DB, ABDO CHN, et al. Epidemiologia da disfunção erétil no Brasil: resultados da pesquisa nacional do projeto avaliar. Revista Brasileira de Medicina, 2004; 61(9):613-25.

23. MYSKOW L. Perimenopausal issues in sexuality. Sexual and Relationship Therapy, 2002; 17(3):253-60.

24. NAEINIAN MR, SHAEIRI MR, HOSSEINI FS. General health and quality of life in patients with sexual dysfunctions. Urology Journal, 2011; 8(2):127-31.

25. NELAS P, COUTINHO E, CHAVES C, et al. Motivação para ter sexo em estudantes do ensino superior. International Journal of Developmental and Educational Psychology, 2018; 2(1):45-54.

26. PECHORRO PS, ALMEIDA Al, FIGUEREDO CS, et al. Validação portuguesa da nova escala de satisfação sexual (NSSS). Revista Internacional de Andrología, 2014; 13(2):47-53.

27. PENTEADO SRL, FONSECA AM, BAGNOLI VR, et al. Avaliação da capacidade orgástica em mulheres na pós menopausa. Revista Associação Médica Brasileira, 2004; 50(4):444-50.

28. PINHEIRO TF, COUTO MT, SILVA GSN. Questões da sexualidade masculina na atenção primária à saúde: gênero e medicalização. Interface - Comunic., Saude, Educ, 2011; 15(38):845-58.

29. RUFINO AC, MADEIRO AP, GIRAO MJBC. O ensino da sexualidade nos cursos médicos: a percepção dos estudantes do Piauí. Revista Brasileira de educação médica, 2013; 37(2):178-85.

30. SARRIS AB, NAKAMURA MC, FERNANDES LGR, et al. Fisiopatologia, avaliação e tratamento da disfunção erétil: artigo de revisão. Rev Med São Paulo, 2016; 95(1):18-29. 